\title{
Amplification of the Zeroth Order Mode in Ultra-thin Layers
}

\author{
José M. Llorens*, Jerónimo Buencuerpo, José M. Ripalda \\ and Pablo A. Postigo \\ IMM-Instituto de Microelectrónica de Madrid (CNM-CSIC), Isaac Newton 8, PTM, \\ E-28760 Tres Cantos, Madrid, Spain \\ *Corresponding Author: jose.llorens@csic.es
}

\author{
Received 5 November 2015; Accepted 3 June 2016; \\ Publication 1 August 2016
}

\begin{abstract}
The ultra-thin limit regime is characterized by a structure which at least in one dimension is significantly smaller than the incoming light wavelength. The absorption in this regime is characterized either by very weak absorption or by a broad peak attributed to the zeroth order Fabry-Perot mode. We show that this mode provides a $14.5 \%$ ultimate efficiency in a $25 \mathrm{~nm}$ GaAs slab on a gold substrate. GaAs in air achieves only a $5 \%$ for a $10 \mathrm{~nm}$ slab. Such an amplification of the resonance absorption is attributed to the high losses of the substrate. For very high losses, the zeroth order mode transits from an over-damped regime to an under-damped one.
\end{abstract}

Keywords: Solar cells, absorption, quasinormal modes, optics, optical coating.

\section{Introduction}

Light absorption plays a central role in the design of optoelectronic devices. It is of particular relevance in the case of solar cells, where the photogenerated carriers result from the absorption of the incoming solar light. The most simple physical model to describe the absorption in the active material of a solar cell is a free-standing slab. The absorption of a slab presents a series of peaks

Journal of Green Engineering, Vol. 5, 71-82.

doi: 10.13052/jge1904-4720.5345

(c) 2016 River Publishers. All rights reserved. 
which are well described by the Fabry-Perot resonances resulting from the interference of the transmitted and reflected waves at the bounding surfaces. Such resonances are commonly described as an integer multiple of the optical path: $m \lambda_{m} / 2=n L$, being $\lambda_{m}$ the wavelength of the resonance of order $m=1,2, \ldots, n$ the refractive index and $L$ the thickness of the slab. The positions of these resonances coincide with the transmission maxima of the slab and conversely, with the minima in the reflectance. If the slab presents optical losses, i.e. its material is described by a complex refractive index, the position of the absorption maxima are slightly shifted with respect to the aforementioned thumb rule of the FP resonances. A broader perspective of the problem is attained from a guidance point of view. If we look at the slab as a two dimensional waveguide, there would be guided modes propagating within the slab at particular frequencies and wavevector orientations thanks to the total internal reflection (TIR) at the top and bottom interfaces [1]. For an incoming angle larger that the TIR critical angle, part of the energy of the field is radiated away from the waveguide, becoming a leaky mode. In a seminal work [2-4], Kliewer, Fuchs and Pardee described such modes as virtualmodes. The particular case of light impinging on a surface at a normal angle is equivalent to the study of a guided mode propagating with a wavevector normal to the waveguide surfaces. Physical methods have evolved in the description of open cavities, being the theory of quasi-normal modes (QNM) the most complete nowadays [5, 6]. Hence, the resonances of an optical system, which are traditionally determined by the poles of the scattering matrix [7], are the eigenvalues of the QNM allowing a rigorous description of optical systems like photonic bandgap structures [8,9] and plasmonic cavities [10].

In the ultra-thin regime $(L / \lambda \ll 1)$ an additional peak can be identified which has no correspondence with any order $m$, as illustrated in Figure 1. In the same plot, we show how the real part of the QNMs eigenfrequencies $\left[\operatorname{Re}\left(\tilde{\omega}_{m}\right)\right]$ points perfectly the peak absorption maxima, while the simple interference conditions formulated above $\left(\omega_{m}\right)$ appear to be red-shifted. Relying on the QNM theory, we managed to attribute the left-most absorption peak to the excitation of the QNM of zeroth order, i.e. $m=0$ [11]. More intriguing is the fact that the position of this peak is predicted by the imaginary part of the QNMs eigenfrequency $\left[\operatorname{Im}\left(\tilde{\omega}_{m}\right)\right]$. This is attributed to the over-damped nature of the zeroth oder mode, i.e. $\operatorname{Im}\left(\tilde{\omega}_{m}\right) \gg \operatorname{Re}\left(\tilde{\omega}_{m}\right)$, as discussed in [11].

In the current study, we analyze the impact of the optical properties of the substrate on the features of the absorption peak. We will see that the presence of a metallic substrate amplifies enormously the absorption of the zeroth order 


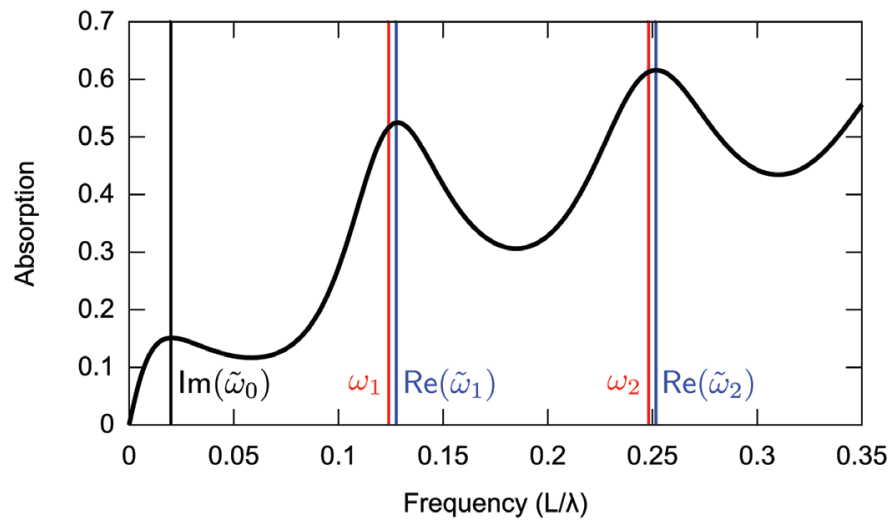

Figure 1 Optical absorption for a free-standing slab of refractive index $N=4.0+i 0.35$. The vertical lines denote the position of the resonances as predicted by the real (blue) and imaginary (black) part of the eigenfrequecies of the QNMs $\left(\tilde{\omega}_{m}\right)$ and the FP interference condition (red).

model. Indeed, Massiot el al. reported in [12] an experimental absorption of $\approx 70 \%$ at $\approx 650 \mathrm{~nm}$ for a single GaAs layer of $25 \mathrm{~nm}$ on Au substrate, which was identified as an "unexpected" FP resonance [13]. We have identified such peak with the zero-frequency mode of the structure and studied the critical role played by the losses of the substrate. The absorption in ultra-thin films might be enhanced even further by introducing a diffraction grating [14], being even possible to achieve the total light absorption in a narrow band [15].

\section{Optical Absorption in the GaAs/Au System}

In the quest for achieving ultra-thin optoelectronic devices, Massiot et al. proposed in [12] an absorber design where the active region consists of a single $25 \mathrm{~nm} \mathrm{GaAs}$ layer finalized with a silver nano-grid embedded in a $\mathrm{SiN}_{x}$ matrix. In Figure 5 of [12] the theoretical and experimental absorption of the isolated GaAs layer on the Au substrate are reported. Both curves show a very good agreement. To get an insight in the performance of just an ultra-thin slab of GaAs in a solar cell, we show in Figure 2(a) the total absorption together with the absorption in the semiconductor to identify the amount of light absorbed in GaAs from that absorbed in the Au substrate. The values of the dielectric function for GaAs and $\mathrm{Au}$ are taken from [16] and [17], respectively. Even at the extreme narrowness of the GaAs slabs considered in the calculations, the amount of absorbed light is very significant. This point is better illustrated in Figure 2(b) by the ultimate efficiency, which is computed as: 


$$
\eta_{\mathrm{ult}}=\frac{\int_{\lambda_{\min }}^{\lambda_{\max }} I(\lambda) A(\lambda) \lambda / \lambda_{\max } d \lambda}{\int_{0}^{\lambda_{\max }} I(\lambda) d \lambda},
$$

being $I(\lambda)$ the AM 1.5 solar radiation spectrum, $A(\lambda)$ the GaAs layer absorption, $\lambda_{\min }=350 \mathrm{~nm}$ and $\lambda_{\max }=872 \mathrm{~nm}$ (the GaAs bandgap at $300 \mathrm{~K}$ ). The top bound of $\eta_{\text {ult }}(A(\lambda)=1)$ for these settings is $45 \%$. A clear maximum can be identified at $25 \mathrm{~nm}$. At larger thicknesses the absorption peak overlaps the GaAs bandgap region, which acts as a high-pass filter, hence resulting in a less efficient absorption. The value of the maximum $\eta_{\text {ult }}$ is $14.5 \%$, meaning that with such a small amount of GaAs a third of the theoretical ultimate efficiency is achieved. The amplification effect can be quantified by comparing this value with that obtained in a free-standing GaAs slab. It is possible to see in Figure 2(b) that the first maximum in $\eta_{\mathrm{ult}}$ reaches a $5 \%$ at $\mathrm{L}=10 \mathrm{~nm}$. Hence, the gold substrate plays a crucial role in the amplification of the zeroth order resonance. This result might be interpreted as an effect of a better overlapping
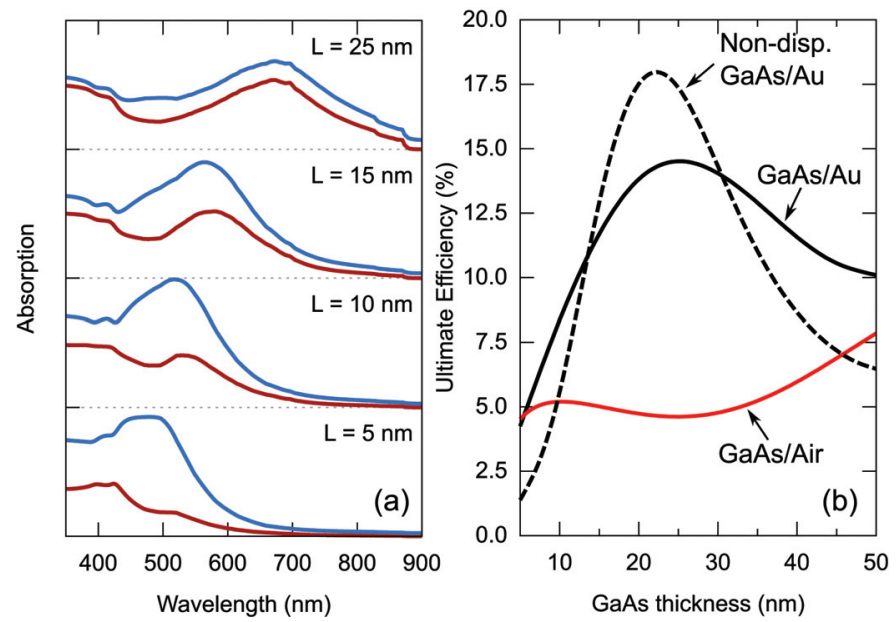

Figure 2 (a) Total absorption (blue) and absorption in the GaAs layer (red) for different slab thicknesses. The spectra are displaced vertically one to each other. The dotted horizontal lines denote the different axis scale from zero to one. (b) Ultimate efficiency taking into account only the absorption in GaAs on Au for dispersive (solid black) and non-dispersive materials (dashed black, $N_{\mathrm{GaAs}}=3.83+i 0.15$ and $N_{\mathrm{Au}}=0.155+i 3.6$ ). Dispersive GaAs on air case (solid red). 
between the mode and the absorbing material. As the metallic substrate is a better back-reflector, the mode tail does not penetrate so deeply into the back material and experiences a confinement in the active region. The total absorption increases even further due to the losses present now in the substrate. These losses play a little effect in our study, because we have considered only the absorption in the active layer along the paper. In general, the enhancement of the zeroth mode absorption increases with a higher refractive index contrast and a higher absorption in the substrate. The latter effect is discussed in the next section.

The position of the maximum is difficult to predict because of the dispersion of the materials. A direct search of the resonance position requires a location of the poles of the scattering matrix in the complex plane. Therefore, an analytical continuation of the dielectric function of the involved materials must be available. For the sake of simplicity, we follow a different approach. The dielectric response of GaAs and Au is described by a fixed value provided that the same qualitative results to those reported in Figure 2 are obtained. Taking as a reference the value of the refractive index at the position of the maximum for $L=25 \mathrm{~nm}(650 \mathrm{~nm})$, we use the following values $N_{\mathrm{Au}}=0.155+i 3.6$ and $N_{\mathrm{GaAs}}=3.83+i 0.15$ to describe the materials. The resulting $\eta_{\text {ult }}$ is shown in Figure 2(b) to facilitate the comparison. As can be appreciated in the plot, both curves show a qualitative good agreement. The absorption spectrum is shown in Figure 3 in dimensionless units given

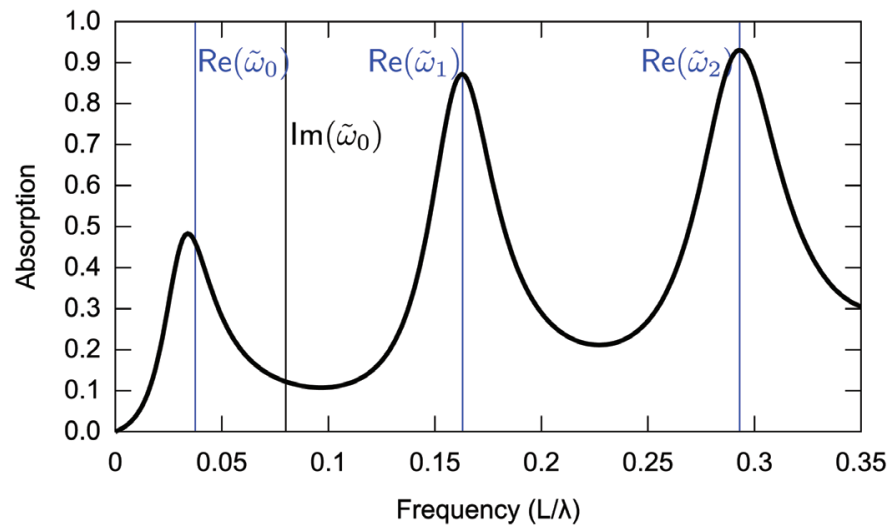

Figure 3 Absorption spectrum for a slab of index $N=3.83+i 0.15$ on a substrate of index $N=0.155+i 3.6$. The vertical lines denote the position of the resonances as predicted by the real (blue) and imaginary (black) part of the eigenfrequencies of the QNMs $\left(\tilde{\omega}_{m}\right)$. 
that the absence of material dispersion permits to apply the scale invariance of Maxwell's equations. From the comparison with Figure 1, it is remarkable the change in the shape of the spectrum. Now the absorption is more intense and the peaks have experienced a reduction of their linewidth. Such change is even more pronounced for the zeroth order mode. Instead of appearing as a "shoulder", it adopts a Lorentzian shape, like any other FP resonances. This effect is again related with the presence of a substrate which acts as a back reflector.

The position of the resonances can be inferred from the eigenfrequencies of the QNMs [11]:

$$
\tilde{\omega}_{m}=\frac{m}{2 N_{\mathrm{GaAs}}}+\frac{i}{4 \pi N_{\mathrm{GaAs}}} \ln \left\{\left(\frac{1-N_{\mathrm{GaAs}}}{1+N_{\mathrm{GaAs}}}\right)\left(\frac{N_{\mathrm{Au}}-N_{\mathrm{GaAs}}}{N_{\mathrm{Au}}+N_{\mathrm{GaAs}}}\right)\right\} .
$$

The vertical lines in Figure 3 help to localize their position in the spectrum. As we saw in Figure 1 the position of the first and second order FP peaks is well predicted by the real part of $\tilde{\omega}_{m}$. However the position of the zeroth order is now better predicted by the real part than the imaginary part. The metallic substrate induces a strong blue shift of the eigenfrequency. The resonances lose their over-damped character becoming critically-damped, i.e. $\operatorname{Im}\left(\tilde{\omega}_{m}\right) \approx$ $\operatorname{Re}\left(\tilde{\omega}_{m}\right)$. Therefore the position of the maximum is neither the imaginary nor the real part of the eigenfrequency.

\section{Effect of the Substrate Losses on the Zeroth Order Resonance}

The fact of replacing the air (Figure 1) by a metal (Figure 3) as back material results in a huge blue shift of the zeroth order mode. Such strong effect is attributed to the high losses of the substrate. This is illustrated in Figure 4. We have considered two different cases: a substrate defined by $\operatorname{Re}(N)=1.5$ (a) and $\operatorname{Re}(N)=0.155$ (b) and a varying $\operatorname{Im}(N)$. The former case resembles a dielectric material, while the latter is representative of a metal substrate. In both cases, the evolution of the spectra with the imaginary part shows the strong blue shift and the increase of the absorption at resonance. From the comparison can be also noted that the metallic substrate is more efficient in amplifying the FP resonances, in particular that attributed to the zeroth order mode. These results also show that the peak identified in Figure 2 is originated by the zeroth order mode. It is possible to recognize an enhancement in the 

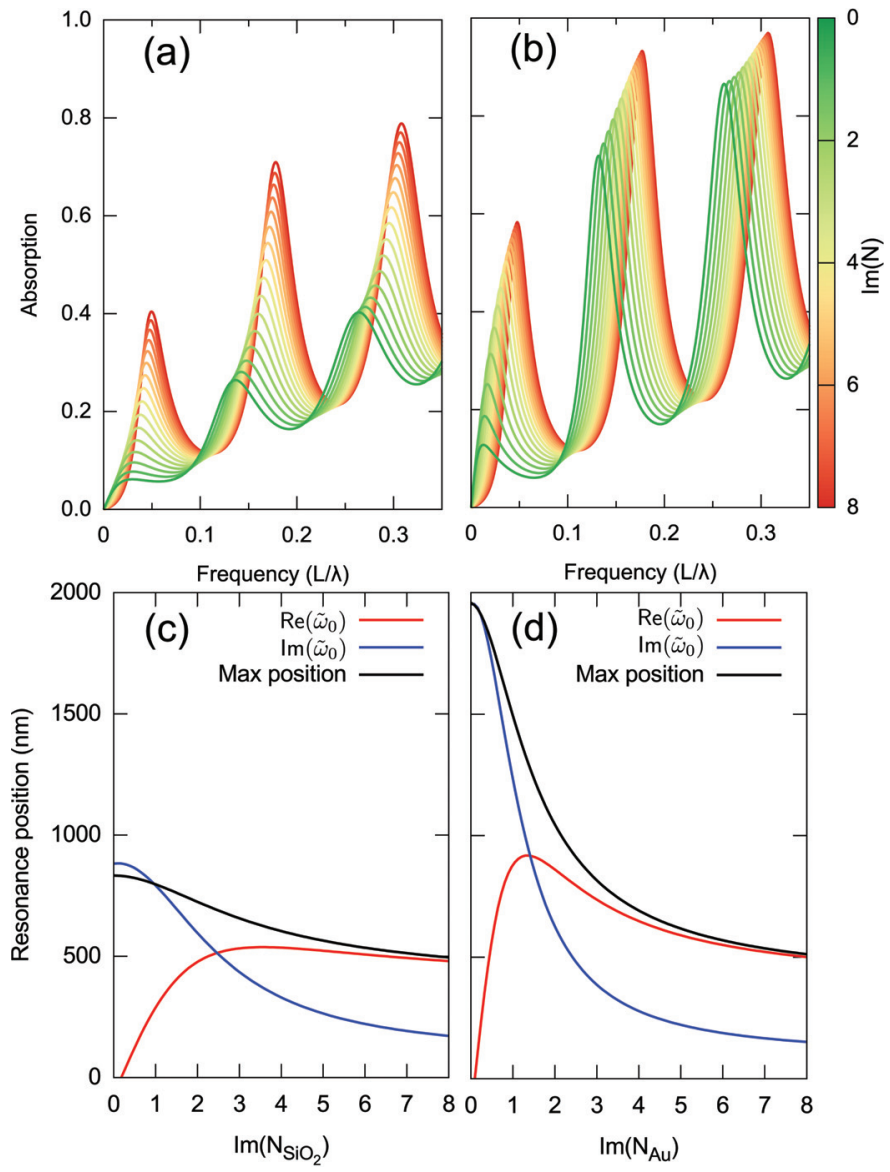

Figure 4 Absorption spectrum for a slab of index $N=3.83+i 0.15$ on a substrate of real index $N=1.5$ (a) and $N=0.155$ (b) for different values of the imaginary part. Position of the absorption maximum of the zeroth order mode compared to the real and imaginary part of the resonance for the same slab of $L=25 \mathrm{~nm}$ on a substrate of real index $N=1.5$ (c) and $N=0.155$ (d) and different values of the imaginary part.

light trapping as a result of having a higher index contrast in the metallic case than in the dielectric one.

The change in shape of the zeroth order peak evolves from that of an over-damped resonance to the case of a critically-damped and finally an under-damped one. It is possible now to study the evolution of the peak maximum as a function of the substrate losses. In Figure 4(c) and (d), the position of such maximum is extracted by inspection of the absorption 
spectra for the two aforementioned cases. The peak position is expressed in wavelength terms to facilitate the comparison. For negligible losses, the imaginary part of the eigenfrequency is significantly larger than the real part, which corresponds to the over-damped case. Indeed, the imaginary part provides an accurate estimate of the position of the maximum for both types of substrates. However, as the losses increase, the position of the maximum deviates from the imaginary part and slowly converges to the real part of the eigenfrequency. Such convergence is achieved in the under-damped case.

\section{Conclusions}

We have studied the absorption of slabs in the ultra-thin regime, meaning thicknesses of a few tens of nanometers. These systems are very interesting for solar cells [12] and also for optical coatings [18]. Relying on the theory of quasi-normal modes, we have successfully attributed the absorption peak appearing in a $25 \mathrm{~nm} \mathrm{GaAs}$ slab on Au to the excitation of the zeroth order mode. We have also shown that the losses of the substrate play an important role in the amplification and shift towards optical wavelengths of the zeroth order resonance. In the past, the origin of such resonance was qualitatively explained by means of strong interference effects $[12,18]$. In this work, we have provided a complementary description solely based on the quasi-normal modes of the structure.

\section{Acknowledgments}

We acknowledge the financial support through projects TEC2015-64189-C32-R and PCIN-2013-179 (MINECO/FEDER), AIC-B-2011-0806 (MINECO), S2013/MAE-2780 (Community of Madrid) and by the European Space Agency contract No 4000112049/14/NL/MV under the GSP/ARIADNA program.

\section{References}

[1] Allan W. Snyder and John D. Love. Optical Waveguide Theory. Chapman \& Hall, 1991.

[2] K. L. Kliewer and R. Fuchs. Optical modes of vibration in an ionic crystal slab including retardation. I. Nonradiative region. Phys. Rev, 144 (2):495-503, 1966. 
[3] K. L. Kliewer and R. Fuchs. Optical modes of vibration in an ionic crystal slab including retardation. II. Radiative region. Phys. Rev., 150(2):573-588, 1966.

[4] R. Fuchs, K. L. Kliewer, and W. J. Pardee. Optical properties of an ionic crystal slab. Phy. Rev., 150(2):589-596, 1966.

[5] E. S. C. Ching, P. T. Leung, A. Maassen van den Brink, W. M. Suen, S. S. Tong, and K. Young. Quasinormal-mode expansion for waves in open systems. Reviews of Modern Physics, 70(4):1545-1554, 1998.

[6] P. T. Kristensen and S. Hughes. Modes and mode volumes of leaky optical cavities and plasmonic nanoresonators. ACS Photonics, 1(1):2-10, 2014.

[7] E. Popov. II Light Diffraction by Relife Gratings: A Macroscopic and Microscopic View. In E. Wolf, editor, Prog. Optics, Vol. 31, pp. 139-187. Elsevier, 1993.

[8] A. Settimi, S. Severini, N. Mattiucci, C. Sibilia, M. Centini, G. D'Aguanno, M. Bertolotti, M. Scalora, M. Bloemer, and C. M. Bowden. Quasinormal-mode description of waves in one-dimensional photonic crystals. Physical Review E, 68(2):026614, 2003.

[9] A. Settimi, S. Severini, and B. J. Hoenders. Quasi-normal-modes description of transmission properties for photonic bandgap structures. J Opt. Soc. Am. B, 26(4):876, 2009.

[10] Q. Bai, M. Perrin, C. Sauvan, J.-P. Hugonin, and P. Lalanne. Efficient and intuitive method for the analysis of light scattering by a resonant nanostructure. Optics Express, 21(22):27371-27382, November 2013.

[11] J. M. Llorens, J. Buencuerpo, and P. A. Postigo. Absorption features of the zero frequency mode in an ultra-thin slab. Applied Physics Letters, 105(23):231115, 2014.

[12] I. Massiot, N. Vandamme, N. Bardou, C. Dupuis, A. Lemaître, J.-F. Guillemoles, and S. Collin. Metal Nanogrid for Broadband Multiresonant Light-Harvesting in Ultrathin GaAs Layers. ACS Photonics, 1:878-884, 2014.

[13] N. Vandamme, I. Massiot, C. Colin, A. Cattoni, N. Bardou, C. Sauvan, P. Lalanne, J. F. Guillemoles, and S. Collin. Plasmonic Nano-Gratings for High-Efficient Ultrathin GaAs Solar Cells. In 28th European Photovoltaic Solar Energy Conference and Exhibition, 2013.

[14] Björn C. P. Sturmberg, Kokou B. Dossou, Lindsay C. Botten, Ross C. McPhedran, and C. Martijn de Sterke. Fano resonances of dielectric gratings: symmetries and broadband filtering. Optics Express, 23(24): A1672, 2015. 
[15] Björn C. P. Sturmberg, Teck K. Chong, Duk-Yong Choi, Thomas P. White, Lindsay C. Botten, Kokou B. Dossou, Christopher G. Poulton, Kylie R. Catchpole, Ross C. McPhedran, and C. Martijn de Sterke. Total absorption of visible light in ultrathin weakly absorbing semiconductor gratings. Optica, 3(6):556, June 2016.

[16] E. D. Palik. Handbook of Optical Constants of Solids. Academic Press, Burlington, 1997.

[17] P. B. Johnson and R. W. Christy. Optical Constants of the Noble Metals. Physical Review B, 6(12):4370-4379, 1972.

[18] M. A. Kats, R. Blanchard, P. Genevet, and F. Capasso. Nanometre optical coatings based on strong interference effects in highly absorbing media. Nature Materials, 12(1):20-24, January 2013.

\section{Biographies}

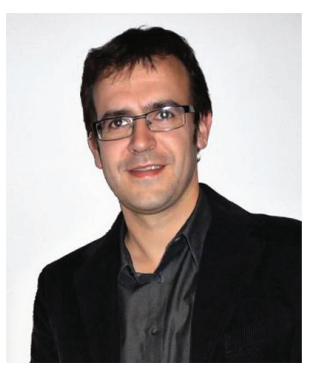

J. M. Llorens born in Valencia (1976). He received his degree (1999) and Ph.D. (2007) in Physics from the University of Valencia. Post-doc from 2007 to 2009 in the Advanced Concepts Team of ESA in the Netherlands. Since 2009 he is working at the Institute of Microelectronics of Madrid CSIC. His research interests are in the electronic structure of semiconductor nanostructures and nanophotonics based on them. He is active in design of lasers, LEDs and solar cells. 


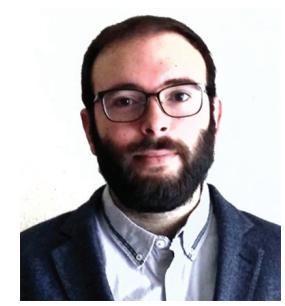

J. Buencuerpo born in Madrid (1986). He received his degree (2010) in Physics and Master in Applied Physics (2011) from the University Complutense of Madrid. Since 2012 he is working at the Institute of Microelectronics of Madrid (CSIC) as Ph.D. student. His research interests are in light trapping designs using nanophotonic crystals and the electronic response of these structures for solar applications. He is active in design and fabrication of nanostructured solar cells.

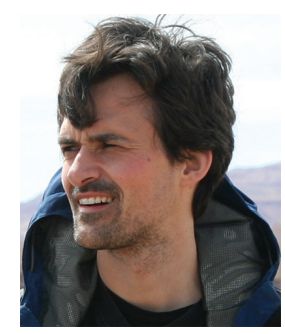

J. M. Ripalda born in Bochum (1972). He received his degree (1995) and Ph.D. (2001) in Physics from the Universidad Autonoma de Madrid. Post-doc from 2001 to 2003 in Imperial College London. Since 2003 he is working at the Institute of Microelectronics of Madrid CSIC. His research interests are in III-V semiconductor technology and photovoltaics. Currently he is most active in the design of concentrator solar cells (CPV). 


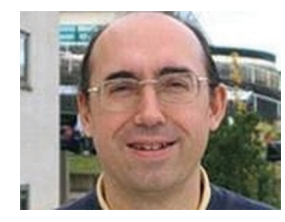

P. A. Postigo received his M.Sc. degree in Physics from the University of Basque Country in 1992, and the Ph.D. degree in Physics from the Polytechnic University of Madrid in 1996. He was Postdoctoral Associate at the Department of Electrical Engineering of the Massachusetts Institute of Technology (MIT) where he worked in optoelectronic integration of lasers on GaAsVLSI chips. He is permanent researcher at the Instituto de Microelectronica de Madrid (IMM-CNM-CSIC) in Spain since 2005. His work is focused on the design, fabrication and characterization of nanophotonic devices including photonic crystal lasers, single-photon emitters and photonic crystal solar cells. 